\title{
Discussion on the effect of improved method in otomycosis with pevisone
}

\author{
shaoli Wang ${ }^{1}$, guoqing Deng ${ }^{1}$, zhibin Zhao ${ }^{1}$, zheng $\mathrm{Fu}^{1}$, xin $\mathrm{Wei}^{1}$, and sheng $\mathrm{Xu}^{2}$ \\ ${ }^{1}$ Hainan Provincial People's Hospital \\ ${ }^{2}$ Maternal and Child Health Hospital of Hubei Province
}

June 15, 2020

\begin{abstract}
Objective: To discuss the effect of improved method in otomycosis with pevisone. Method: 160 patients of otomycosis were randomly divided into traditional group (TG) and improved group (IG) equally. All the patients, external auditory canals (EACs) were cleaned by the cerumen hook and attractor, in TG, pevisone was applied by traditional method after cleaning the EACs, but in IG, the cotton balls were used to clean the EACs additionally and pevisone was applied by our improved method. The treatment was implemented once every other day for three times in total in two groups. At 3d, 1wk, 1mo, $6 \mathrm{mo}$ of treatment, cure rates were analyzed and compared between the two groups,at $6 \mathrm{mo}$ of treatment the recurrence rate was compared between the two groups. Results: The cure rates in the IG(50\%,66.7\%,61.5\%,53.8\%) were higher than that in the $\mathrm{TG}(32.9 \%, 48.7 \%, 46.1 \%, 34.2 \%)$ at four observation time points respectively,the difference between the two groups were statistically significant $(\mathrm{p}<0.05)$. The recurrence rate in IG was lower than which in TG $(9 \% \mathrm{vs} 22.4 \%)$ at 6 mo of treatment $(\mathrm{p}<0.05)$. Conclusion: The effect of improved method in otomycosis with pevisone has great advantages both in the therapeutic effect and treating process, which is worth further promoting in clinical practice.
\end{abstract}

\section{Objectives}

Otomycosis is a sub-acute or chronic epithelial inflammatory disease caused by fungal infection in theexternal auditory canal(EAC), which is acommon disease in the otorhinolaryngology clinic and usually caused by species of Candida and Aspergillus ${ }^{1-3}$. At present, the widely used therapeuticdrugsfor otomycosisare antifungal drugs,pevisoneand other topical drug preparations ${ }^{4-6}$.Since 2011, we started to clean up the EAC and applypevisoneto cover the EAC for treating otomycosis with the help of otoendoscopy, most patients have been treated satisfactorily, now the treatment method has been developed for a long time and improved.In order to discuss the effect of improved methodin otomycosis with pevisone, we report the results as follows.

\section{Design}

160 patients who diagnosed otomycosis from out-patient department were selected as subjects, they were divided into traditional group (TG) and improved group (IG) by random number table method equally.The treatment was implemented once every other day for three times in total in two groups. At $3 \mathrm{~d}, 1 \mathrm{wk}, 1 \mathrm{mo}$, 6 mo of treatment, cure rates were analyzed and compared between the two groups, at 6 mo of treatment the recurrence rate was compared between the two groups. At the follow-up time points, if cure criteria were met, use only the drug.Safety was evaluated by recording any adverse events.

Traditional method : soaked the EAC with $3 \%$ hydrogen peroxide for a few minutes. Under the otoendoscopy, the mildew moss, attachment or secretions were removed by the cerumen hook and attractor. Then covered the surface of EAC and tympanic membrane with pevisone by a cotton swab(Figure1 A) 
Improved method : specially processed cotton balls were used compared to the traditional method. After the soaking and cleaning step, the cotton batting of a cotton swab was taken down and shaped into two small cotton balls, inserted the tail end of the cotton swab into one cotton ball and pushed it till reached the tympanic membrane surface and then rotated it, the lesions attached to the surface of EAC which could not be cleaned by the cerumen hook and attractor could be all wiped down by the cotton ball, the remaining hydrogen peroxide could also be absorbed away. Then sent the second cotton ball which fully mixed with pevisone to the tympanic membrane like before, rotated it outward until the surface of EAC was covered with pevisone uniformly, when the cotton ball tightly wrapped around the tail of the cotton swab after rotation, they could be extracted out together. (Figure1 B、C、D、E)

\section{Settings}

Clinical manifestations : itching, otorrhea,ear fullness, hearing impairment, tingling, tinnitus, etc ${ }^{7-8}$. Otoendoscopy examination shows grayish white-brownish yellow - black membranous mildew moss, which sometimes exists as powdery, granular attachment, when being cleaned up, congestive and swelling EAC skin can be seen, sometimes with mild erosion, when tympanic membrane is involved, congestion or rough surface could be seen on it.

Etiological examination (fungal smear test) : mildew moss, attachment or secretions were taken for test. Diagnosis was confirmed when hyphae and/or spores were seen under microscope.

Efficacy evaluation criteria ${ }^{9}$ : Cure: symptom-free and had no abnormal secretions in the EAC determined by otoendoscopy, the fungal smear test is negative; Improved: the clinical symptoms alleviated, the fungal smear test is positive or negative; Recurrence: the same or similar clinical symptoms occur again after one month of treatment, confirmed by the positive fungal smear test.

Statistical method : Statistical analyses were performed using SPSS for Windows

version 17.0 (SPSS). The measurement data were expressed by $(\mathrm{x} \pm \mathrm{s}) \cdot \chi^{2}$ test was used for statistical interpretation. The $\mathrm{p}$ values below 0.05 were considered significant.

\section{Participants}

From January 2017to October 2019,160 patientswho diagnosed otomycosis from out-patient department were selected as subjects.Written consent was obtained from each patient before including him in the study. Local ethics committee had approved the study.Inclusion criteria:(1) Diagnosed with otomycosis: visualization compatible with fungal debris is done through microscopic examination;(2)Cooperate with the doctor's treatment and follow up on time. Exclusion criteria:(1)Pregnant and lactating women, patients who used other therapies or treatment, diseases or structural anomalies impeding therapeutic response evaluation;(2)Malignant invasive (acute or chronic) otitis externa complicated by mastoiditis or meningitis, or both.

\section{Main outcome measures}

\section{General information}

The traditional group (TG) had 41 males and 35 females, aged 19.2-66.7 years, with an average of $44.5 \pm$ 6.2 years, whose disease course was $0.5-11.6$ weeks, with an average of $2.5 \pm 0.4$ weeks, while the improved group (IG) 0had 46 males and 32 females aged 18.2 to 65.8 years, average age $43.3 \pm 5.7$ years, whose disease course was 0.6-12.3 weeks, with an average of $2.2 \pm 0.3$ weeks(Table1); there was no significant difference in age and sex composition between the two groups $(P<0.05)$. There were 6 patients withdrawn, 4 from the TG ( 2 lost to follow up, 2 due to noncompliance with the treatment plan), and 2 from the IG (lost to follow up). Among the ultimate 154 subjects, diagnoses were made in 73 of the left ear, 61 of the right and 20 of both sides,according to the same treatment, the poorer therapeutic effect of the ear was included into the study results for patients with binaural otomycosis. No adverse reaction in all subjects. The main symptoms were ear itching $(87.7 \%)$, ear fullness $(82.5 \%)$,otorrhea(78.6\%), hearing impairment $(64.3 \%)$, tingling $(41.6 \%)$, tinnitus $(26 \%)$ (Figure 2). 


\section{Comparison of therapeutic effects between the two groups}

For the improved group(IG), symptoms improved rate was $90 \%$ after the first treatment, with a $50 \%$ cure rate at the same time. At the 1 week and 1 month follow-up, the cure rates were $66.7 \%$ and $61.5 \%$ respectively. 7 cases recurred at 6 months, with a cure rate of $53.8 \%$. For the traditional group(TG), symptoms improved rate was $50 \%$ at the first visit, while the cure rate was only $32.9 \%$. At the 1 week and 1 month follow-up, the cure rates were $48.7 \%$ and $46.1 \%$ respectively. At the 6 months follow-up, 17 cases recurred, with a cure rate of $34.2 \%$. The cure rates of the IG were significantly higher than that in the TG at the four observation time points respectively, the difference between the two groups was statistically significant $(\mathrm{P}<0.05)$. The therapeutic effects of pevisone in the two groups are shown in Table 2.

\section{Pictures of the two treatment method results in typical cases}

Otoendoscopic images of the two groups of typical cases at 1week of treatment are shown in Figure 3. As can be seen, the pathology of patients in the IG improved significantly,no fungal secretions were found in the EAC by otoendoscopy examination; while in the patients of the TG, there were still some scattered lesions at the same time point.

\section{Results}

The incidence ofotomycosisrelates to the injury of $\mathrm{EAC}^{10-11}$, which caused by the use of hard materialsorviolent manipulation to the ears; the cross-infection due to ear cleaning in public places such as barber shops, or swimming in potentially contaminated water, chronic diseases impairing the immunity like diabetes mellitus ${ }^{12}$ and the increase in the use of antibiotics and hormones ${ }^{11}{ }^{13}$, in addition, the climate of Hainan island is hot and humid all year round,otomycosis is particularly frequent in this kind of regions ${ }^{14-16}$. The common strains have been reported in the literatures ${ }^{12}{ }^{17}$ that the Candida and Aspergillusspecies were the most common agents, whichare sensitive to antifungal agents.

Pevisone mainly consists of $0.1 \%$ triamcinolone acetonide and $1 \%$ econazole. Triamcinolone acetonide is a medium-effective glucocorticoid hormone, which can improve the anti-allergic, anti-exudation, antiinflammatory and anti-pruritic effects ${ }^{18}$. Econazole is a derivative of imidazole which has a broad-spectrum antifungal effect, and is also effective for Gram-positive bacteria ${ }^{19}$. So as a compound preparation the pevisone has multiple advantages of anti-allergic, anti-inflammatory, anti-pruritic and antifungal effects, meanwhile it's safe reliable and rapid-onset.

The most widely used treatment regimen for otomycosis includes mechanical debridement of the ear canal along with local antifungal agents, however, there are still problemssuch as recurrence orresidual disease ${ }^{10}$, and unsatisfactory cure rate,heavy pain during the treating process etc. We found some clinical difficulties in our practice, at first, the remaining mildew moss and purulent secretions couldn't be cleaned up thoroughly, which may impact the effect of drugs or lead to recurrence. Secondly, when we cleaned the lower anterior part of EAC and the surface of tympanic membrane, it was hard for patients to cooperate with the doctor because of the pain, which led to incomplete cleaning of the lesion. Thirdly, for patients with narrow or over curved EAC, it was difficult to clean up the deep partowing to the limited visual field, especially the lower anterior part, where required the assistance of cotton applicators or alligator forceps for cleaning, which were costly in China. In addition, when we covered the surface of EAC with pevisone by a cotton swab, the head of cotton swab was too large to reach tympanic membrane,and it was impossible to confirm that all the surface of EAC were covered with pevisone. We found that in the literatures there was a lack of specific treatment skill for removing the lesion, and no reference to the difficulty in the treating process and how to relieve pain.

In order to solve the problems above, we improved the procedure during treating process. The cotton balls + the cotton swabtail in the improved method has the following advantages: 1.Soft cotton balls scraping can lower the risk of injuries to tympanic membrane, in contrary, the metal suction device can cause injuries when doing the cleaning work on the tympanic membrane; in addition, cotton balls can relieve the pain in the treating process;2. The suction device can only suck up the lumpy lesions, but the debris or mud-like 
lesions attached to the surface of EAC can't be completely removed. The rotation of the cotton ballswhich acts like brushing can thoroughly clean the surface of EAC and absorb away the remaining liquid at the same time;3.The suction device can hardly reach the lower anterior part of EAC where the lesions are usually residual, but the cotton swab tail can easily send the cotton ballthere; 4 . Compared to the big head of the cotton swabs, the cotton ball is much smaller, which makesthe visual field is clear relatively,sothe EAC can be cleaned thoroughly anddrug can be covered uniformly with the cotton ball; 5.Instead of the costly cotton applicators or alligator forceps, a cheap cotton swab is competent.

In the literatures the frequency and course of treatment for otomycosis are still uncertain.In our study,the treatment frequency was once every other day for three times in total, the treatment course ended within one week, and we choose the $3^{\text {rd }}$ day, the $1^{\text {st }}$ week, the $1^{\text {st }}$ month and $6^{\text {th }}$ month of treatment as the observation time points. The cure rates of the improved group(IG)were higher than that in the traditional group(TG) at four observation time points respectively $(\mathrm{P}<0.05)$. At $6 \mathrm{mo}$ of treatment, the recurrence rate of the IG was lower than that of the TG, the difference was statistically significant $(\mathrm{P}<0.05)$, which proved that the cure rate and recurrence rate can be significantly improved with the improved method compared to the traditional method. In addition,we can see the cure number and proportion showed a downward trend from the $1^{\text {st }}$ week,that may means the treatment course and frequency need to be further studied.

\section{Conclusions}

The effect of improved methodin otomycosis with pevisonehas great advantages both in the therapeutic effect and treating process, which is worth further promoting in clinical practice.

Figure 1.A:in traditional method, covered the surface of EAC with pevisone by a cotton swab;B:in improved method, the cotton batting was shaped into two balls;C: one of the cotton balls was mix with pevisone;D: the cotton ball was sent to the tympanic membrane surface with thethe tail end of the cotton swab;E: the surface of EAC was covered with pevisone uniformly.

Figure 3: A、B: the same case in the improved group. A shows the membranous mildew moss tightly attached to the surface of EAC and the tympanic membrane,B shows no fungal secretions were found in the EACat 1week of treatment;C、D: the same case in the traditional group. C shows the large grayish-white mildew lump and purulent secretions in EAC when included,D showsthere were still some scattered lesions in the EAC at 1week of treatment.

\section{References}

1.S.C. Prasad, S. Kotigadde, M. Shekhar, et al.Primary otomycosis in the Indian subcontinent: predisposing factors, microbiology, and classification.Int J Microbiol, 2014 (2014), pp. 1-9

2.F. Khan, R. Muhammad, M.R. Khan, et al. Efficacy of topical clotrimazole in treatment of otomycosis.J Ayub Med Coll Abbottabad, 25 (2013), pp. 78-80

3.H. Nowrozi, F.D. Arabi, H.G. Mehraban, et al.Mycological and clinical study of otomycosis in Tehran, Iran.Bull Environ Pharmacol Life Sci, 3 (2014), pp. 29-31

4.Moharram AM, Ahmed HE, Nasr SAM: Otomycosis in Assiut, Egypt. J Basic Appl Mycol 4, 1-11 (2013)

5.Anwar K, Gohar MS: Otomycosis; clinical features, predisposing factors and treatment implications. Pak J Med Sci 30, 564-567 (2014)

6.De la Paz Cota BR, Cepero Vega PP. Efficacy and safety of eberconazole 1\% otic solution compared to clotrimazole 1\% solution in patients with otomycosis.Am J Otolaryngol. 2018 May - Jun;39(3):307-312.

7.B. Barati, S. Okhovvat, A. Goljanian, et al.Otomycosis in central Iran: a clinical and mycological study.Iran Red Crescent Med J, 13 (2011), pp. 873-876

8.T. Ho, J. Vraben, D. Yoo, N. Coker. Otomycosis: clinical features and treatment implications. Otolaryngol Head Neck Surg, 135 (2006), pp. 787-791 
9.Peng YX, Diagnosis and Treatment of Otitis Externa Mycotica: Experiences with 216 Cases. Chinese Journal of Otology Vo1.17,No.4 , 2019

10. Jia X, Liang Q, Chi F, et al. Otomycosis in Shanghai: Aetiology, clinical features and therapy. Mycoses 55, 404-409 (2012)

11.Abdelazeem M, Gamea A, Mubarak H, et al. Epidemiology, causative agents, and risk factors affecting human otomycosis infections. Turk J Med Sci 45, 820-826 (2015)

12. Mohammed Rifaat Ahmed, Ashraf Saad Abou-Halawa, Waheed F. Hessam,et al.A search for new otomycotic species and their sensitivity to different antifungals.Interv Med Appl Sci. 2018 Sep; 10(3): 145-149.

13.Agarwal P, Devi L S. Otomycosis in a Rural Community Attending a Tertiary Care Hospital: Assessment of Risk Factors and Identification of Fungal and Bacterial Agents. J Clin Diagn Res. 2017;11(06):DC14DC18.

14.A.A. Malik, S.N. Malik, M.A. Aslam, et al. Comparative efficacy of topical clotrimazole and 3\% salicylic acid in otomycosis.Rawal Med J, 13 (2012), pp. 46-49

15. Fasunla J, Ibekwe T, Onakoya P. Otomycosis in western Nigeria.Mycoses. 2008;51(1):67-70.

16.C. Linstrom, F. Lucente.Diseasesof the external ear.J. Bailey, T. Johnson, D. Newlands (Eds.), Head\& neck surgery-otolaryngology, Lippincott Williams \& Wilkins, Philadelphia,PA (2014), pp. 1987-2002

17.Satish H S, Viswanatha B, Manjuladevi M. A Clinical Study of Otomycosis. IOSR J Dental Med Sci. 2013;5(02):57-62.

18.Li Y, He L. Diagnosis and treatment of otomycosis in southern China.Mycoses. 2019 Nov;62(11):10641068.

19. Ren Y, Zhang Q, Yu Z, et al. Clinical analysis of external ear canal coating by otoendoscopy on otomycosis external with triamcinolone acetonide and econazole nitrate cream. Lin Chung Er Bi Yan HouTou Jing Wai Ke Za Zhi. 2015;29:1304-1305.

\section{Hosted file}

Figure.docx available at https://authorea.com/users/333431/articles/459644-discussion-onthe-effect-of-improved-method-in-otomycosis-with-pevisone

\section{Hosted file}

Table.docx available at https://authorea.com/users/333431/articles/459644-discussion-on-theeffect-of-improved-method-in-otomycosis-with-pevisone 